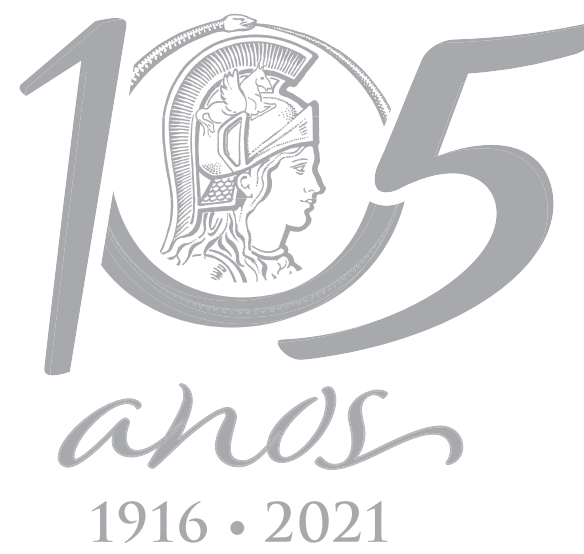

\title{
Evaluation of the protocol for thirst management using ice popsicles in the immediate postoperative period: A pilot study in southern Brazilian hospital
}

\author{
DENISE M. TEREZA, GABRIELA M. BALDASSO, RODRIGO S. PAES, MARIA E.J. \\ PACHECO, PATRÍCIA P.S. ROSA BEATRIZ VENDRAMINE, SERGIO C. HULSE, \\ RAFFAELE CAPASSO \& RAFAEL C. DUTRA
}

\begin{abstract}
Although a protocol for immediate postoperative thirst management has been applied clinically in a discrete way, most surgical and nursing teams remain neglected, unlike other complications in the postoperative period. This pilot study aimed to demonstrate if the ice popsicles promote thirst satiety and subsequently investigate the mechanism involved in this process. Twenty-five volunteers in the immediate postoperative period were recruited to participate in this study conducted in a tertiary medical center in southern Brazil. In this protocol, ice popsicles decreased the intensity and discomfort of thirst. Relevantly, all patients (100\%) demonstrated satiety after ice popsicles administrated in the immediate postoperative period. Altogether, ice popsicles constitute a promising, innovative, and simple strategy for the management of thirst, mainly after surgery, adding to patient comfort and the security of the health team that manages it.
\end{abstract}

Key words: Clinical signals, ice popsicles, postoperative, thirst.

\section{INTRODUCTION}

Water comprises over $80 \%$ of the human brain, cardiovascular tissue, skeletal muscle, kidney tissue and gastrointestinal tissue (Wang et al. 1999). Thus, water has an essential role in the metabolism of the human body, substrate transport across membranes, cellular homeostasis, temperature regulation, and circulatory function (Armstrong \& Johnson 2018). In this context, an important mechanism related to the maintenance of water balance is the sensation of thirst, which in turn triggers the consumption of water or other fluids (Ramsay \& Booth 1991, Arai et al. 2013, Labbe et al. 2009).

Thirst is a primordial emotion that motivates fluid intake to compensate for constant water loss incurred from breathing, sweating, and urine production. However, thirst is not the only mechanism related to water balance. It comprises the sensing and integrating of different signals related to heart rate, blood $\mathrm{CO}_{2}$ levels, temperature, inflammation, and the homeostatic response of thirst (Ramsay \& Booth 1991, Critchley \& Harrison 2013, Gogolla 2017). Some theories approach thirst as an interaction between physiological, psychological, and environmental inputs to the brain (Armstrong \& Kavouras 2019). Nonetheless, the non-homeostatic thirst is directly correlated with water intake. It is determined by the taste and temperature of a fluid, the rhythmicity of drinking, feeding 
schedules, and drinking behavior (Thompson et al. 1991, Armstrong \& Kavouras 2019). Moreover, dehydration promotes changes in intracellular and extracellular volume, namely osmotic and hypovolemic loss that result in thirst activation, respectively. Hypovolemic thirst occurs when baroreceptors detect decreased blood volume and release angiotensin II, which consequently promotes the secretion of aldosterone to retain sodium $\left(\mathrm{Na}^{+}\right)$and conserve water. On the other hand, in osmotic thirst, plasma hyperosmolarity (especially related to $\mathrm{Na}^{+}$levels) causes intracellular water to be transported to the blood, initiating the dehydration of supraoptic neuronal cells. It stimulates the secretion of antidiuretic hormone (ADH) (also called arginine vasopressin [AVP] or simply vasopressin), which acts immediately on the kidneys to enhance the reabsorption of water to dilute the increased osmolality (Arai et al. 2014, Bury \& Rosseneu 1985). Consequently, a $\mathrm{Na}^{+}$level $>145 \mathrm{mEq} / \mathrm{L}$ is defined as hypernatremia and commonly results from a water deficit, inadequate intake of water or impaired thirst mechanisms. Concomitantly, 7\% of intensive care unit (ICU) patients experience hypernatremia (Caballero et al. 2005). However, a decrease of approximately $10 \%$ of the plasma volume (Caballero et al. 2005, Agrawal et al. 2008) is required to initiate hypovolemic thirst. When extracellular fluid volume depletion is extreme (e.g., > 10\% loss of body mass), physiological compensation includes vigorous drinking (Cheuvront \& Kenefick 2014) and increased $\mathrm{Na}^{+}$consumption (Ramsay \& Booth 1991) The fluid replacement is a physiological stimulus considered a conscious aspect of the regulation of blood volume. In this way, the motivation to drink is mostly autonomous and objective the homeostatic normalization of intra- and extracellular volume (Armstrong \& Kavouras 2019). The satiety of thirst begins before the body absorbs fluids, starting this way already during drinking. It is assumed that the preabsorptive mechanism has the ability to quench thirst through awareness of osmoreceptors and volume receptors in the mouth and throat, which trigger the signal to stop drinking through interrupting the release of ADH (Arai et al. 2014, Garcia et al. 2019, Saltmarsh 2001). Also, the wetting of the mouth by drinking and an increase of saliva secretion play a role in quenching thirst (Eccles 2000, Peyrot des Gachons et al. 2016). The stimulus promoted in the mouth through the activation of osmoreceptors located in the gastrointestinal tract provokes an answer from specialized sensory cells positioned in the brain's preoptic/hypothalamic region (Aloamaka et al. 2018). Additionally, previous studies have identified primary sensory neurons and pathways that can drive homeostatic forms of thirst, as well as water intake that occurs in anticipation of an impending deficit (Bourque 2008) Relevantly, decreases in blood volume can promote homeostatic thirst through the activation of different brain regions, including: i) the nucleus tractus solitarius and parabrachial nucleus (McKinley \& Johnson 2004); ii) subfornical organ (SFO) neurons in response to increased concentrations of circulating angiotensin II (Fitzsimons 1998, Zimmerman et al. 2016); iii) organum vasculosum lamina terminalis, which drives homeostatic water intake in response to systemic hypertonicity (Bourque 2008); and iv) anterior cingulate cortex and insula, which seem to be involved in the perception of thirst (Saker et al. 2014). In the anticipatory process, an important function was noted with SFO neurons, especially the GABAergic population, which suppresses drinking. These neurons analyze the level of physiological need, and the amount of water recently ingested, predicting homeostasis restoration. The same can be observed in the $\mathrm{MnPO}$ neuron activity. The neural activity declines through water intake, demonstrating real-time 
feedback between interoceptive gastric inputs or oropharyngeal inputs and neuron activity. Also, the inhibition of glutamatergic neurons in the SFO region through cold water ingestion shows that cold liquids are more thirst-quenching (Zimmerman et al. 2017, Gizowski \& Bourque 2017). Surprisingly, it remains unknown whether neurons can encode qualities of emotion that serve to motivate water intake behavior and whether the different stimuli that drive thirst can be integrated at the subcortical level.

In the immediate postoperative period and during post-anesthesia recovery, patients are exposed to several factors predisposing to the symptom of thirst, such as prolonged preoperative fasting, anesthetic-surgical medications, tracheal intubation and/or blood loss (Arai et al. 2013, Tosun et al. 2015). When the confluence of these factors is added to anxiety, irritability, stress and fear of anesthesia and surgery (Tosun et al. 2015, Dessotte et al. 2016, Conchon \& Fonseca 2018), it results in thirst being one of the most distressing aspects for the patient. As demonstrated by Puntillo and colleagues, 70.8\% of ICU patients rated thirst as having the greatest intensity, and it was rated as more intense than fatigue, anxiety, restlessness, hunger, dyspnea, pain, sadness, fear, and confusion (Puntillo et al. 2010). Furthermore, Nascimento and colleagues demonstrated that despite $61.7 \%$ of the patients experiencing intense thirst, the discomfort was not verbalized, showing the weakness in treating this symptom (Nascimento et al. 2019). Even drugs commonly used in the intraoperative period, such as anticholinergics, anesthetics and opioids, are related to the perception of thirst (Arai et al. 2014) A very interesting study conducted by Nascimento and colleagues developed and validated the safety protocol for thirst management during the postoperative period, which used level of consciousness, absence of nausea and vomiting, and reflexes of protection of the airways (cough and swallowing) as safety criteria (Nascimento et al. 2014) Afterwards, other authors used the same protocol successfully (Nascimento et al. 2014, Motta et al. 2020, do Nascimento et al. 2018). Nonetheless, the management of thirst continues to be neglected by surgical and nursing teams, unlike other complications in the immediate postoperative period, such as pain, nausea, and postoperative vomiting, which are permanently identified, monitored and treated.

With regard to this problem, previous reports evaluating different interventions to relieve thirst or dry mouth in the pre- and postoperative periods suggested the effectiveness of menthol moisturizers (Aroni et al. 2020, Eccles 2000, Schafer et al. 1986), ice popsicles (Conchon \& Fonseca 2018, Nascimento et al. 2014, Motta et al. 2020), sprays of cold sterile water from squirt bottles, gargling with cold water and oral swabs with cold sterile water (Cho et al. 2010, Puntillo et al. 2014). Currently, it is known that cold water satiates thirst more effectively than body temperature water (Deaux 1973) and is preferred because it offers greater relief from mouth dryness (Brunstrom 2002). Additionally, cold water also may stimulate greater saliva production than warm water and, in turn, alleviate mouth dryness (Brunstrom \& Macrae 1997), as well as activate thermal receptors in the oropharynx, such as transient receptor potential melastatin 8 (TRPM8) and transient receptor potential ankyrin 1 (TRPA1) (Dhaka et al. 2006). The identification of TRPM8, a menthol- and cold-activated ion channel, demonstrated that different classes of TRP channels are involved in thermosensation (Peier et al. 2002) and thirst satiety (Eccles et al. 2013). TRPM8 was identified by its expression in sensory neurons and its ability to be activated by cold and menthol (Peier et al. 2002). Slight cooling, TRPM8 is directly activated, depolarizing sensory neurons mediated by a 
sizeable enthalpic change that results in the channel opening reaction (Latorre et al. 2011). Interestingly, in accordance with the proposed role of TRPM8 as an innocuous cool receptor, the channel does not colocalize with nociceptive fibers, such as calcitonin gene-related peptide (CGRP), isolectin B4 (IB4), substance P, and TRPV1 (Peier et al. 2002), and whether TRPM8 has a role in cold nociception remains controversial.

Keeping the above data in mind, the purpose of the present study was to demonstrate whether the ice popsicles promotes thirst satiety and clinical signals in the immediate postoperative period in a southern Brazilian hospital. Herein, the pilot of the experimental phase was realized, which investigated the mechanism involved in this process by evaluating ADH levels, hemoglobin, hematocrit, $\mathrm{Na}+$, and $\mathrm{K}+$ levels, urinary osmolarity, and renin serum levels in immediate postoperative patients after received or not receiving ice popsicles.

\section{MATERIALS AND METHODS}

\section{Ethical aspects}

This study was approved by the Research Ethics Committee of the Universidade Federal de Santa Catarina (UFSC), complying with Resolution $466 / 12$ of the National Health Council (research under number CAAE 02779818.4.0000.0121).

\section{Study design, place and period}

This experimental pilot study included 20 patients submitted to elective surgeries at a private tertiary hospital located in the countryside of the state of Santa Catarina with adult patients. The institution has 105 hospital beds and receives municipal and state referrals for procedures of high complexity. The institution has a surgical center with eight operating rooms and 12 post-anesthesia care rooms and performs, on average, 700 surgical procedures per month and 7870 surgical procedures per year. Data collection occurred from January 2019 to March 2019 and included patients aged 17 to 74 years.

\section{Sample and inclusion and exclusion criteria}

The population was composed of adult patients in the immediate postoperative period, and the sample consisted of 25 participants divided into the: i) Control group, which did not receive any treatment after surgery $(n=10)$; ii) Experimental group, which received ice popsicles immediately after surgery $(n=10)$, and iii) Healthy control group, which was not submitted to surgery and not received any intervention ( $n=5)$ (Figure 1).

Inclusion criteria were patients of both sexes, aged between 17 and 74 years, with spontaneous thirst verbalization or, when questioned, with intensity $\geq 3$ on the Numerical Verbal Scale (NVS), with anesthesia duration of $>45$ minutes, assisted in the post-anesthesia recovery room and with approval in the evaluation of the security protocol on thirst management (do Nascimento et al. 2018). Patients with i) swallowing restriction; ii) allergy to ice; iii) continuity lesion(s) in the oral mucosa; iv) cognitive deficits, heart disease, diabetes or using continuous medication; or v) whose anesthetic recovery did not occur in the post-anesthesia recovery room were excluded (Figure 1).

\section{Study protocol}

The primary clinical outcome of interest was comparing the thirst perception in the initial and final surgery moments. After that, the secondary clinical interests were evaluating the satiety reach (zero intensity) in patients submitted to the ice popsicles protocol. Secondly, in the preoperative period, patients who met the eligibility criteria were invited to participate in the study and signed the Informed Consent Form. In the post-anesthesia recovery room, 


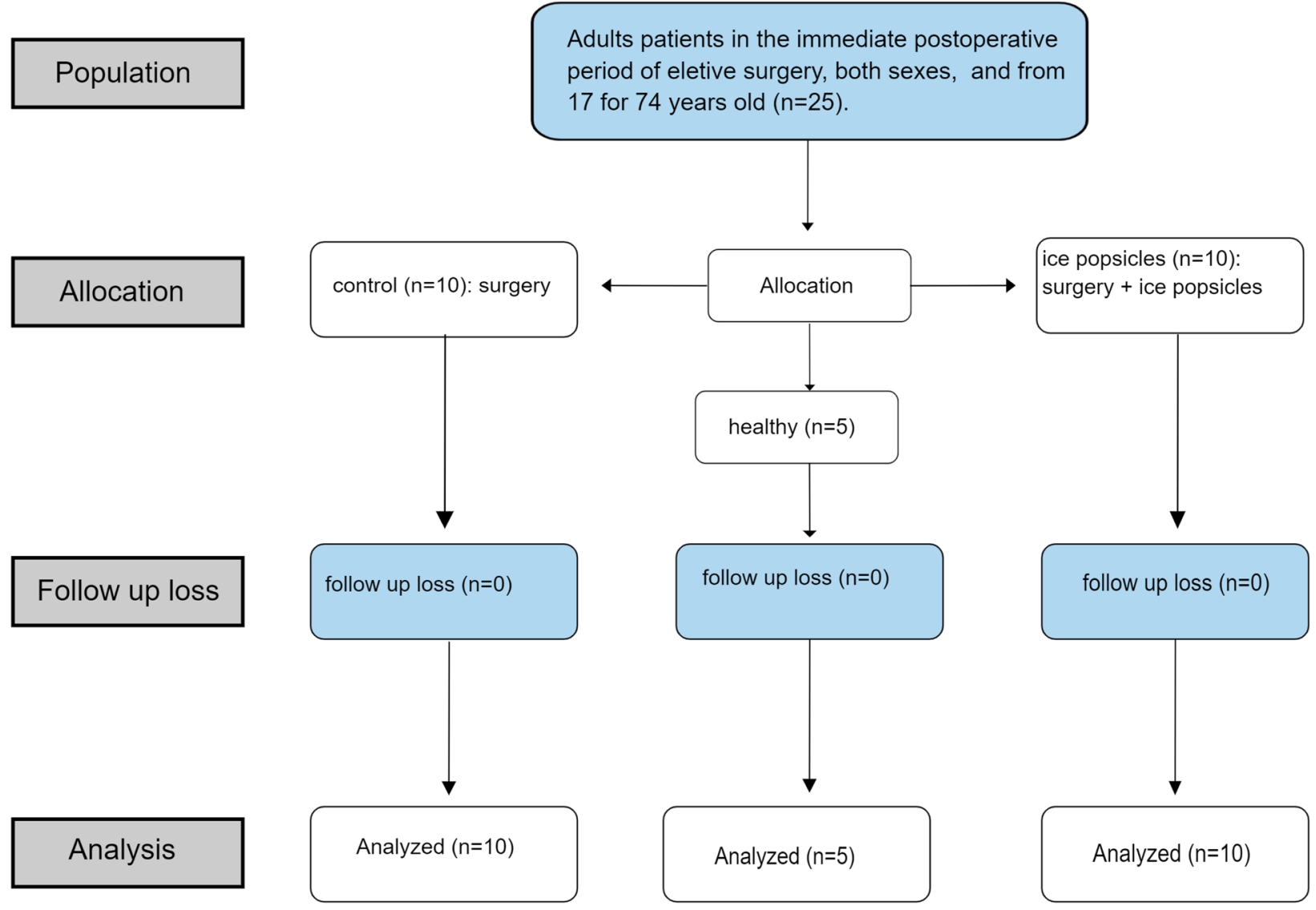

Figure 1. Diagram of sampling and definition of groups.

patients who continued to meet the inclusion despise diuresis immediately after waking up criteria were randomly assigned to the defined group, as previously described. After surgery, in the immediate postoperative period, patients were monitored by the nursing team for the security protocol on thirst management, which evaluated consciousness level, protection of the airways and absence of nausea and vomiting. After approval, the research protocol was initiated (Figure 2).

The ice popsicles were produced in a specific form, with a pre-determined volume of $10 \mathrm{ml}$ and added to the referred hospital's recovery room freezer. The patients received the following guidelines: do not brush the teeth or rinse the mouth on the morning of exam collection (to avoid interference by oropharyngeal stimulation of water and menthol present in toothpaste); in the morning (to avoid false-positive results in relation to nocturnal urinary osmolarity concentrations); abstention from alcohol for 24 hours and exercise 60 minutes before the protocol (as to avoid the interference of physical activity in the release of vasopressin) (Nascimento et al. 2020). Figure 2 shows the flow diagram for the study. The NVS was used to measure the thirst of patients. The scale comprises a score that varies from no thirst (01), light thirst (2-3), moderate thirst (4-6), intense thirst (7-9), and unquenchable thirst (10) (Figure 2d) (Nascimento et al. 2020). The protocol was applied 15 minutes after the patient's arrival in the recovery room. The intervention consisted of a single administration of ice popsicles in the immediate postoperative, considering 
a) Study Design

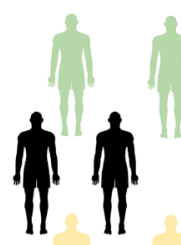

Sample of 25 patients

Mind the

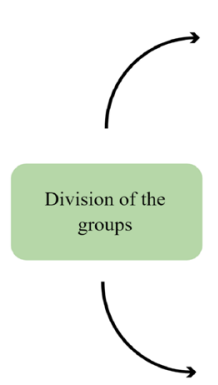

$\underset{\substack{\text { Healthy Control } \\ \text { Group }}}{\left\{\begin{array}{c}\text { Was not submmited } \\ \text { to surgery and not } \\ \text { received any } \\ \text { intervention. }\end{array}\right.}$

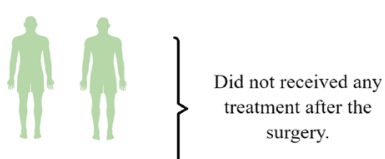

Control Group $\}$ surgery. c)
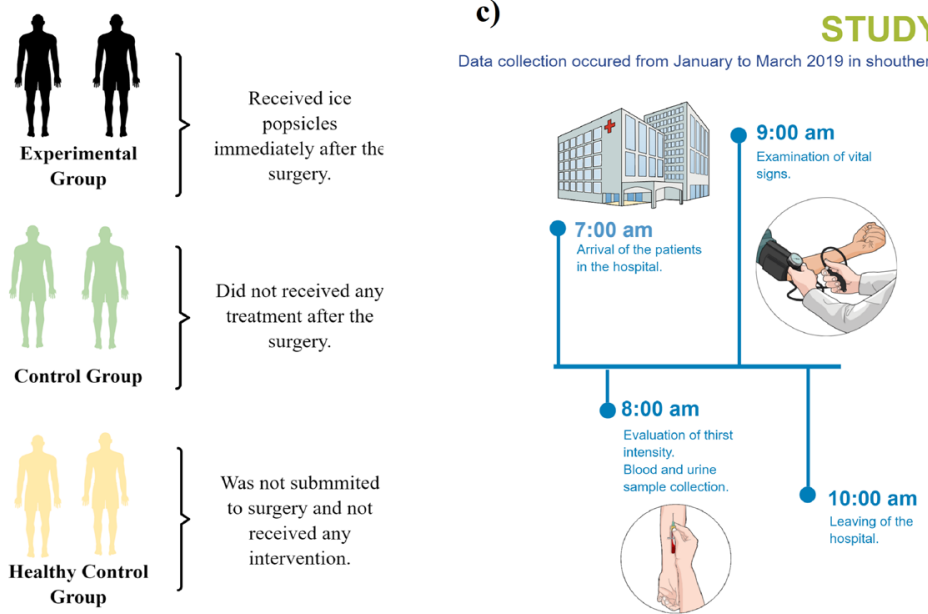

STUDY PROTOCOL

d)

b)

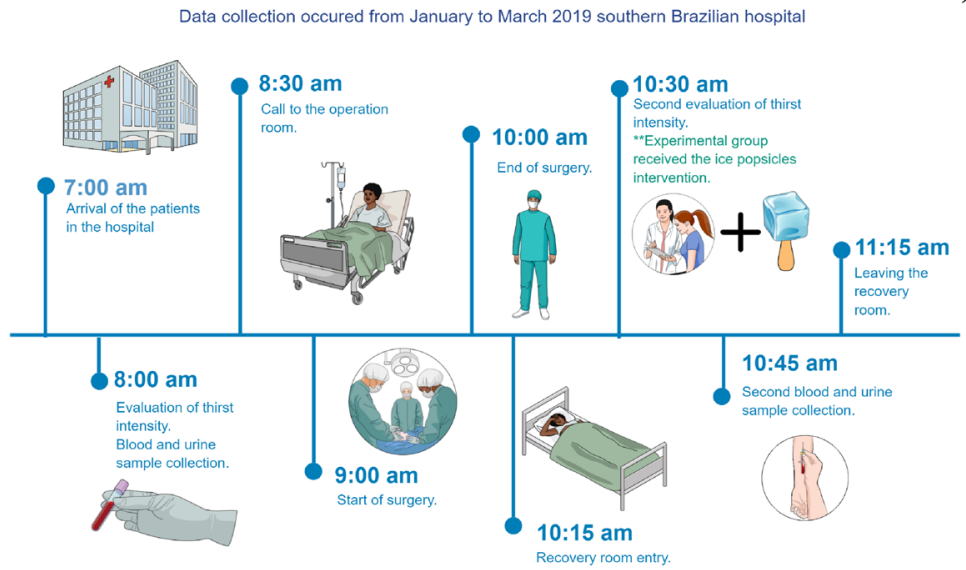

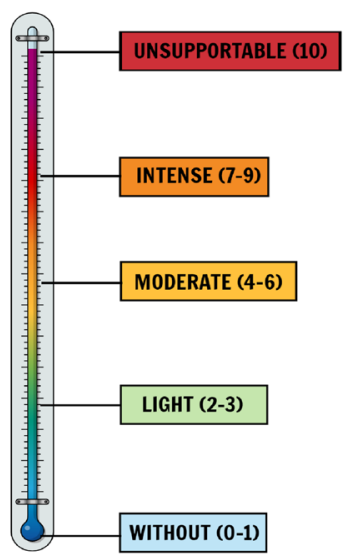

Figure 2. Study design. a) Demonstrates the division of the groups. b) Description of the flow diagram of the study. Initially, the preoperative period included the arrival of the patients to the hospital to collect clinical information and evaluate the thirst intensity. After, in the postoperative period, the application of ice popsicles protocol to a final assessment. c) Demonstrates the flow diagram of the study based on the protocol of the healthy control group. d) The Numerical Verbal Scale (NVS) corresponding to no thirst (0-1), light thirst (2-3), moderate thirst (4-6), intense thirst (7-9), and unquenchable thirst (10). Figure created using the Mind the Graph platform.

that patients already referred to satiety in this administration. The measure of clinical signals and blood collection occurred after 15 minutes of the administration. The participants were evaluated according to the safety criteria, as mentioned above (Figure 2).

\section{Biochemical parameters}

Blood samples were stored in vacuum blood collection tubes containing ethylenediaminetetraacetic acid (EDTA). The serum osmolarity samples were stored in vacutainer tubes with a separating gel. Then, the tubes were identified with the personal data of volunteers and collection times and analyzed in the hospital's laboratory. The vasopressin samples were frozen, while osmolarity samples were refrigerated. Both the hemoglobin and hematocrit samples were analyzed in the Sysmex's Xe2100D equipment (Sysmex, Curitiba, Brazil), which uses a fluorescent flow cytometry methodology. $\mathrm{Na}^{+}$and potassium $\left(\mathrm{K}^{+}\right)$levels 
were analyzed in the Johnson's Vitros FS5600 equipment (Ortho Clinical Diagnostics, New Jersey, USA) through the ion-selective electrode method. Renin levels were detected by the chemiluminescence methodology using Liaison equipment (DiaSorin, Vercelli, Italy). Urine samples were collected in a bottle-type urine collector with a lid and identified with the volunteer's personal data of volunteers and collection times and forwarded to the hospital's laboratory immediately. Urine osmolarity was detected by the cryoscopy method using an advanced osmometer apparatus. Vasopressin was dosed through the radioimmunoassay method, which demonstrates high sensibility and specificity through radiolabeled isotopes. The aim of this methodology was to evaluate the concentrations of unmarked substances through competition with radioisotopelabeled substances. Vasopressin was dosed by radioimmunoassay Bühlmann equipment (Vasopressin Direct RIA, Bühlmann Laboratories AG, Schönenbuch, Switzerland).

\section{Statistical analysis}

The data were analyzed using GraphPad Prism 6 software (GraphPad Software Inc., San Diego, CA, USA). Descriptive variables were described by mean; relative (\%) and absolute frequencies (n); and minimum, maximum and median values. The quantitative data and statistical analysis used the Kolmogorov-Smirnov test, a nonparametric method used to test to two groups. The SidakBonferroni method was performed for the comparisons in multiple tests. Two-way ANOVA was used to grouped analysis (two or more independent variables). P-values $<0.05$ were considered a statistically significant difference.

\section{RESULTS}

This study covered 25 patients divided into different groups (Figure 1). The control group sample was composed of five males (50\%) and five females (50\%), with a median age of 40 years (minimum 19 years and a maximum of 69 years). The fasting time of these patients showed a median of 9 hours and 50 minutes (minimum 2 hours and maximum 16 hours). The most representative surgeries were orthopedic and otorhinolaryngology (30\%), followed by abdominal (20\%), cardiovascular and plastic (10\%). Also, the experimental group sample was composed of four males (40\%) and six females (60\%), with a median age of 34 years (minimum 17 years and maximum 74 years), and ASA I (100\%) composed the sample. The most representative surgeries were otorhinolaryngology (40\%), followed by plastic and neurological (20\%), with median duration of procedure of 1 hour (minimum 10 minutes and maximum 4 hours) (Figure 1 and Table I). Moreover, five females (100\%) composed the sample of the healthy control group, with median age 31 years (minimum 24 years and maximum 41 years) (Figure 1). The healthy control group was not submitted to surgery and not received any intervention. The purpose of this group's inclusion was to evaluate the behavior of the thirst of healthy patients compared with patients submitted to surgery. As observed in Figure 4, a significant difference in this parameter, indicating that the fasting time could interfere in the thirst intensity even in the preoperative period. Also, the inclusion criteria were the same for all the groups, including the healthy. On the other hand, this group's distribution was smaller, considering that the study's principal objective was analyzing the thirst intensity in the immediate postoperative period and not the behavior of thirst. 
Table I. Sociodemographic and clinical characteristics of the participants.

\begin{tabular}{|c|c|c|}
\hline & \multicolumn{2}{|c|}{ Groups } \\
\hline Caracteristics & Control & Ice popsicles protocol \\
\hline Age in years, mean & 31.6 & 37 \\
\hline Median (Min., Max.) & $40(19,69)$ & $34(17,74)$ \\
\hline Sex, $n$ (\%) of males & $5(50)$ & $4(40)$ \\
\hline Type of scheduled surgery, $n(\%)$ & \multicolumn{2}{|c|}{} \\
\hline Plastic & $1(10)$ & $2(20)$ \\
\hline Neurologial & & $2(20)$ \\
\hline Cardiovascular & $1(10)$ & $1(10)$ \\
\hline Orthopedic & $3(30)$ & $1(10)$ \\
\hline Abdominal & $2(20)$ & $4(40)$ \\
\hline Otorhinolaryngology & $3(30)$ & $10: 20$ \\
\hline Fasting time in hours, mean & 9 & $11(6,14)$ \\
\hline Median (Min., Max.) & $9: 50(2,16)$ & $1(0: 10,4)$ \\
\hline Surgery time in hours, mean & $01: 36$ & $10(100)$ \\
\hline Median (Min., Max.) & $1: 20(0: 48,2: 45)$ & \\
\hline ASA physical status calssification, $n(\%)$ & $10(100)$ & \\
\hline ASA I & \multicolumn{2}{|c|}{} \\
\hline
\end{tabular}

Abbreviation: ASA, American Society of Anesthesiologist.

Popsicle administration was applied accordingly the protocol by Conchon and Fonseca (Conchon \& Fonseca 2018), who offered ice popsicles every 15 minutes for 45 minutes to the patients. Our study decreased the administration time to 15 minutes, offering the ice popsicles 15 minutes after the end of surgery (Figures 2 and 3). The patients could control the contact with ice according to their sensibilities, varying the ingestion time from person to person. Furthermore, this time was enough for patients to report thirst satiety. In this way, the experimental group was submitted to the ice popsicles protocol, while the control group did not receive any intervention for thirst management (Figures 2 and 3). Also, the healthy control group did not have a surgical procedure in the last year and did not receive the intervention. As for analyzing the thirst satiety (only immediate postoperative parameters), comparisons were made between the measure in the first 15 minutes after the surgery and the thirst intensity after ice popsicles (Figure 3). Herein, as expected, the difference founded in the analyses was a significant increase in the number of patients referred to thirst-quenching through the ice popsicles protocol. In the first evaluation, one patient (10\%) considered itself satisfied, differently than after the intervention, in which all patients (100\%) demonstrated satiety by administering one dose of $10 \mathrm{ml}$ of ice popsicles (Figure 3).

In another set, interpretations were made to evaluate the thirst behavior (before and after the surgery; Figure $4 a$ and b, respectively). considering the analysis of thirst behavior in 


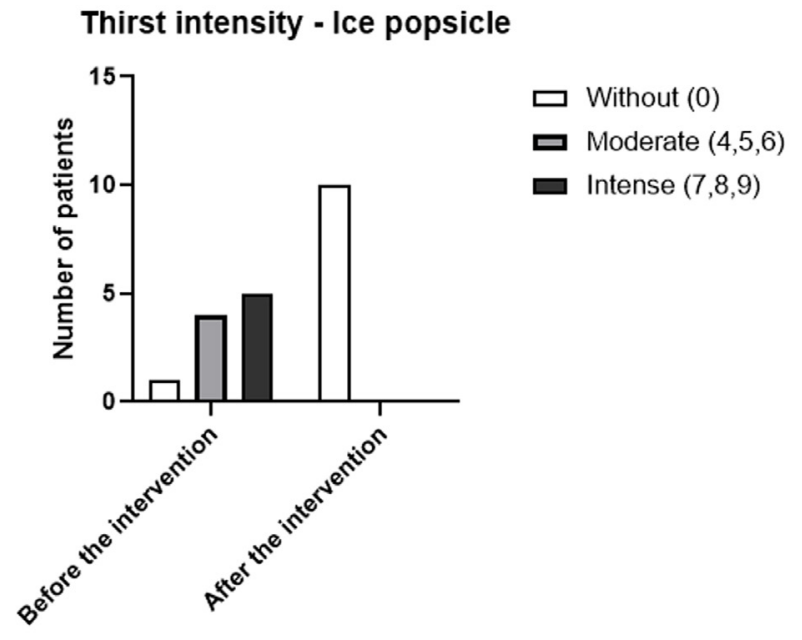

Figure 3. Evaluation of ice popsicles' effectiveness in the intensity of thirst evocated by patients in the immediate postoperative period. Numerical verbal scale (NVS) the intensity of thirst was evaluated in the experimental group before and after the administration of ice popsicles protocol. Data are shown as the mean of five different measurements \pm SD. The statistical analyses were made using column statistics.

the preoperative (Figure $4 \mathrm{a}$ ) and postoperative (Figure 4b) period as expected, three patients (30\%) experienced intense thirst before the surgery, increasing to five patients (50\%) in the postoperative period, during the time the protocol for thirst management with ice popsicles was applied (Figure 4). Herein, the increase in thirst intensity and in the number of patients who experienced intense thirst could demonstrate the thirst behavior during the surgical procedure. On the other hand, the patients who did not report thirst increased from two patients (20\%) to four patients (40\%), demonstrating a reduction in thirst intensity in the control group (Figure 4). Interestingly, the results of hematocrit levels demonstrated a significant difference between the preoperative and postoperative periods when compared to patients in the same group (Figure 5). Additionally, the results of vasopressin (Figure 6a) and renin (Figure 6b) levels illustrated discrete variation between the control group and the group that received ice popsicles, although without a statistically significant difference. Interestingly, after postoperative, the patients treated with ice popsicles had a lesser degree of dehydration as manifested by a higher urine osmolality versus control group (Figure 6c). There was no difference in the absolute level of sodium (Figure $6 \mathrm{~d}$ ) between the individuals treated with ice popsicles and controls. Finally, there are no difference between the absolute level of potassium (Figure 7a) and plasma osmolarity (Figure 7b) in between the control and ice popsicles groups.

\section{DISCUSSION}

This pilot study evaluated an innovative strategy for the relief of thirst during patients' recovery submitted to a surgical procedure, analyzing if the ice popsicles protocol would be an effective alternative. As previously described, during the surgical procedure, the patient is exposed to different factors predisposing to the symptom of thirst, such as fasting time and blood loss (Arai et al. 2013, Tosun et al. 2015), evidencing the importance of devising new strategies for thirst relief and reducing the suffering caused by this discomfort. Until then, the results for thirst intensity were promising, reaching a high percentage of satiety or decreased intensity of thirst, proving the possible effectiveness in the administration of ice popsicles. In view of thirst as a multifactorial symptom, the discomfort caused by it should be considered subjective, evaluating the experience according to the patient's perception (Conchon \& Fonseca 2018, Motta et al. 2020). In this way, the efficacy of the intervention should be evaluated the same way, providing a real judgment about the relief of discomfort. 


\section{Thirst behavior}

a)

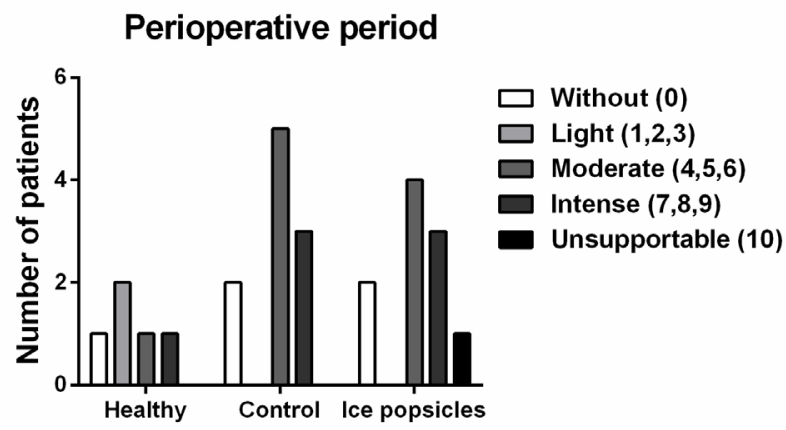

b)

Immediate postoperative period

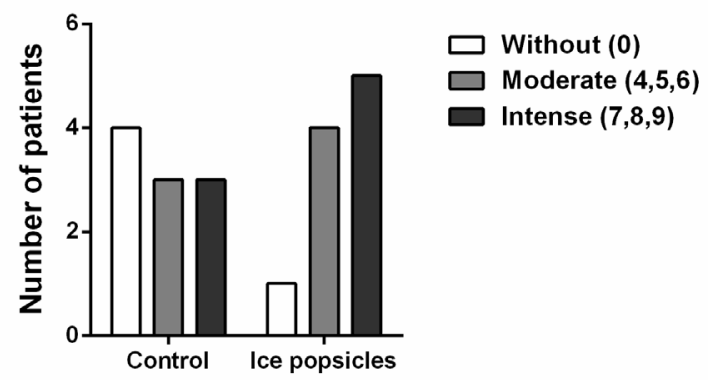

Figure 4. Evaluation of thirst behavior in the comparison between before and after the surgery procedure. Herein, we demonstrated through numerical verbal scale (NVS) the intensity of thirst in the healthy, control, and treated group in the (a) preoperative and (b) immediate postoperative period. Data are shown as the mean of five different measurements \pm SD. The statistical analyses were made using column statistics.

This study's relevance is that it investigates a protocol in the daily practice of management of thirst that considers the patient's experience, not just the predisposition to the symptom of thirst. Therefore, were designed an experimental phase that constituted the evaluation of selected patients in the pre- and postoperative periods.

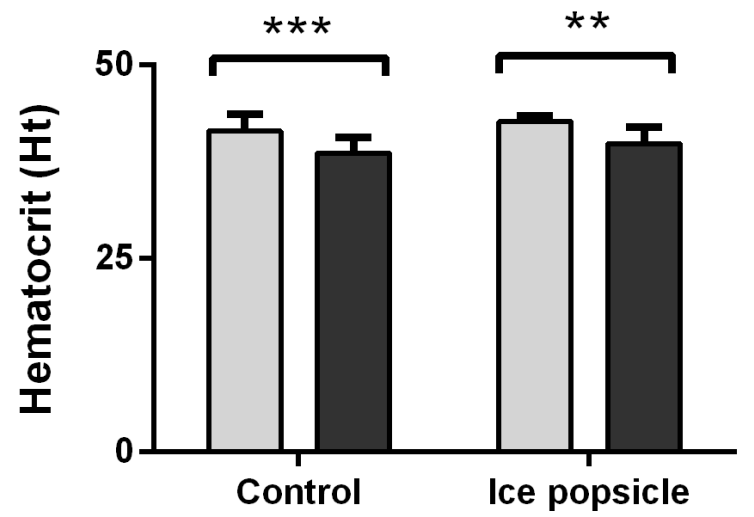

Figure 5. The difference in hematocrit levels between control patients and those submitted to thirst management protocol with ice popsicles. This figure illustrates the difference between hematocrit levels in the preoperative period (light gray) and the postoperative period (dark gray) between healthy, control and treated group. The statistical analysis was made using two-way ANOVA test. *P-values < 0.05 , **P-values $<0.01$ and $* * * P$-values $<0.001$ were considered a statistically significant difference.
This protocol conceived the pilot results. The established increase in thirst intensity during the surgery procedure was observed in the study. Analyzing the behavior of thirst of healthy patients compared with patients submitted to surgery showed a significant difference in this parameter, indicating that the fasting time could interfere in the thirst intensity even in the preoperative period. Patients in the experimental group reported a slight increase in thirst intensity, while patients in the control group exhibited a decrease in the same parameter. Discordant with this, the hematocrit levels of these patients exhibited a considerable statistical difference between the preoperative and postoperative periods. This difference shown between the groups was lower in the experimental group, which received the protocol for thirst management with ice popsicles, suggesting greater dehydration in the control group. Although some authors have demonstrated the intervention effect mediated by the inhibition of vasopressin secretion through the activation of "preabsorptive satiety", providing thirst relief as a response to oropharyngeal stimulation from the cold of the ice popsicles (Puntillo et al. 2014), 
a)

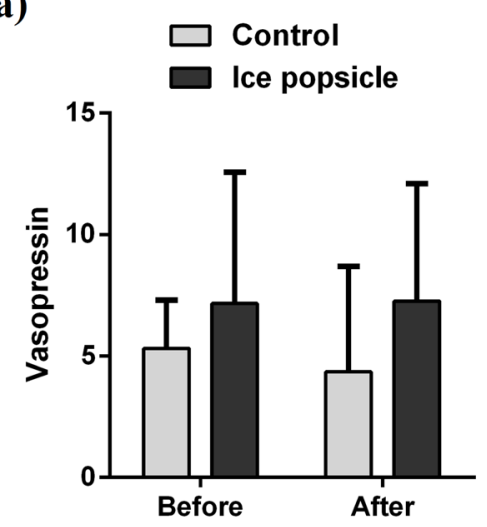

c)

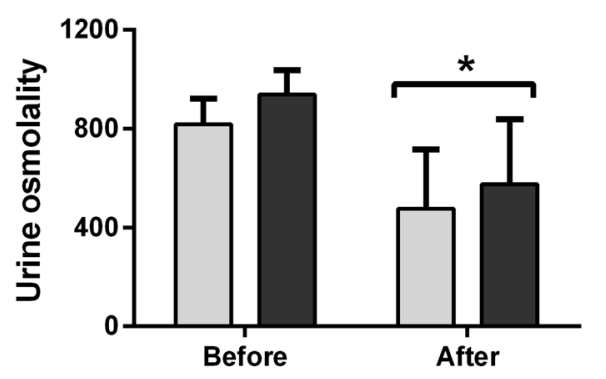

b)

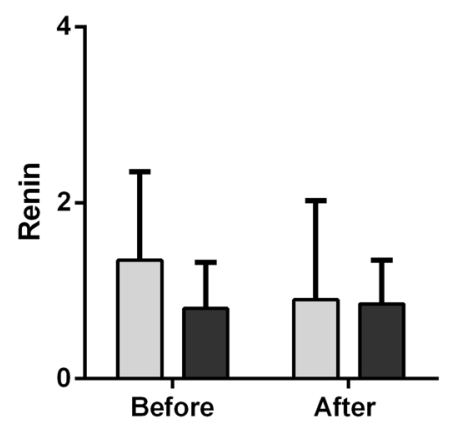

d)

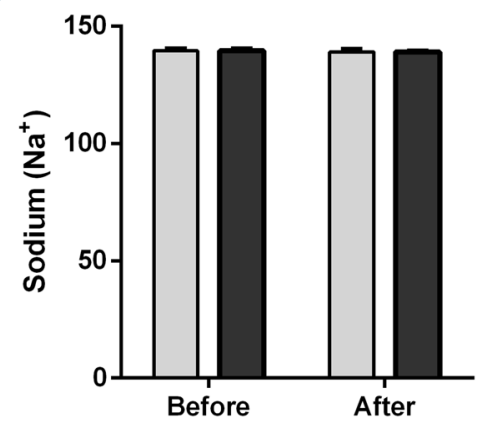

Figure 6. The effects of thirst management using ice popsicles on blood volume and systemic vascular resistance. Herein we demonstrated whether ice popsicles was able to modulate the levels of vasopressin (a), renin (b), urine osmolality (c) and sodium (d). Data are shown as the mean of two different measurements \pm SD. The statistical analysis was made using multiple $t$ test comparisons with Sidak-Bonferroni method. P-values $<0.05$ were considered a statistically significant difference. the vasopressin analysis demonstrated little or no hormonal modification in the groups. The same occurred in the analysis of the renin levels, resulting in non-activation of the hypovolemic mechanism of thirst activation (Caballero et al. 2005, Agrawal et al. 2008). Salata and colleagues demonstrated that oropharyngeal stimulation promoted by ice provoked a rapid decrease in plasma vasopressin levels, even before a change in plasma osmolarity (Salata et al. 1987). In agreement, the differences observed in these parameters could demonstrate that the experimental group suffers a vasopressin inhibition as a response to ice popsicles administration. In contrast, the control group demonstrated an increase in these levels, suggesting more significant dehydration in this group.

Nonetheless, understanding the neural mechanism involved in preabsorptive satiety could help adopt new techniques to alleviate the discomfort. Considering that patients reported satiety in the first 15 minutes of the protocol and that the homeostatic imbalance could be regulated before water consumption through an anticipatory response promoted by the SFO (Zimmerman et al. 2016), quenching of thirst probably occurred before the water absorption and changes promoted in the composition of blood. Also, the hormonal response produced by secretion of renin and vasopressin was slow, while the regulation of drinking behavior was extremely fast. The ending of the protocol before 45 minutes may have interfered with the renin and vasopressin results due to a slow hormonal response. Additionally, neuronal projections related to thirst control are extremely complex and have been explored by comparing pharmacologically induced states of dehydration with satiety. For instance, Denton 
a)

\section{$\square$ Control $\square$ Ice popsicle}

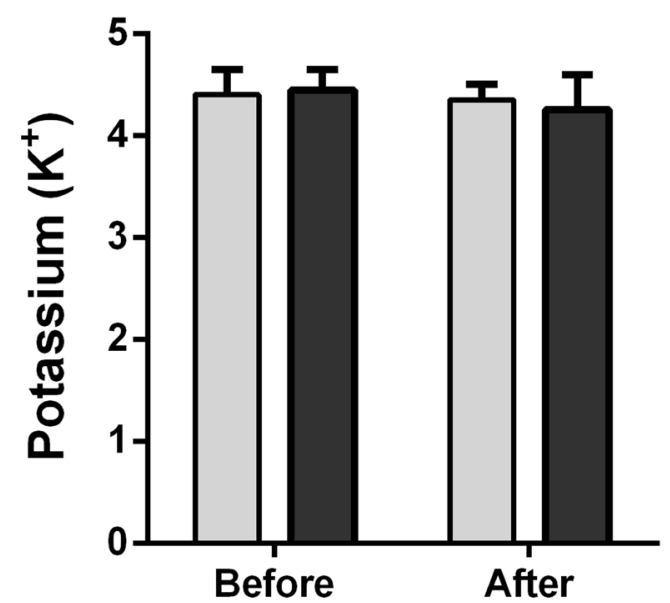

b)

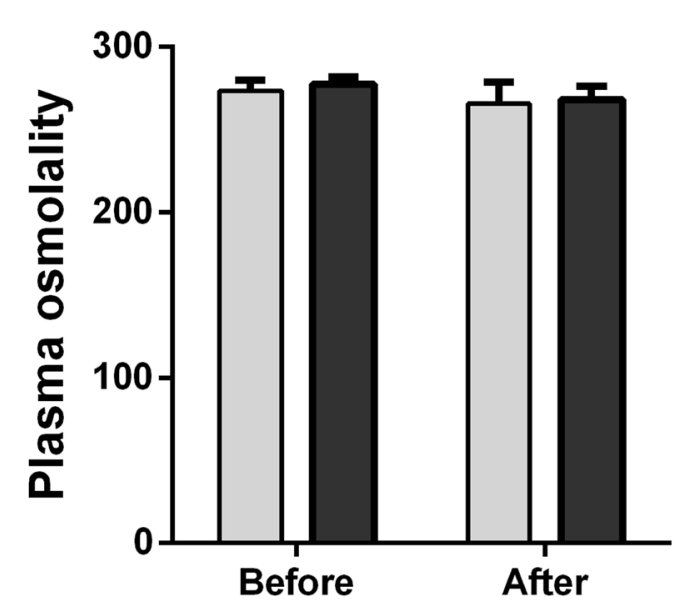

Figure 7. The levels of potassium and plasma osmolality before and post ice popsicles administration. The statistical analysis was made using two-way ANOVA test.

and colleagues demonstrated the correlation of regional cerebral blood flow with thirst score in the posterior cingulate region. Interestingly, cingulate site activation (Brodmann's areas 32, 24, and 31) disappeared immediately after drinking to satiation, demonstrating an important role in the consciousness of thirst. The same authors suggested that a prototypical primal vegetative emotion, such as thirst, represents a "dynamic core" through a complex pattern of activations and deactivations that are functionally integrated, but anatomically distributed (Denton et al. 1999), although several avenues remain to be explored, for instance, if neurons from subcortical level could control the emotion that serves to motivate water intake behavior. Collectively, the results in this study are consonant with the concept of thirst related by a complex pattern of activation and deactivation as a primitive vegetative function, particularly with functional changes in the ancient brain areas, and additional studies will improve our understanding of this essential primordial emotion.

The positive effect observed and the experimental group's consequent total satiety possibly occurred by oropharyngeal stimulation from ice popsicles, as demonstrated by Salata et al. (1987). Also, the small volume associated with ice popsicles $(10 \mathrm{ml})$ demonstrated effectiveness respecting the maximum gastric volume of $50 \mathrm{ml}$ and reducing the risk associated with bronchoaspiration (Perlas et al. 2011). This preliminary evidence suggests a considerable capability of ice popsicles in the promotion of thirst relief and corroborates with Conchon and Fonseca's finding, which demonstrate the effectiveness of $37.8 \%$ of ice popsicles when compared with water at room temperature (Conchon \& Fonseca 2018).

Some limitations of this study need to be considered, including lack the blinding of patients and the main investigator. Thirst discomfort is considered subjective, and the lack of blinding could interfere in the patient's 


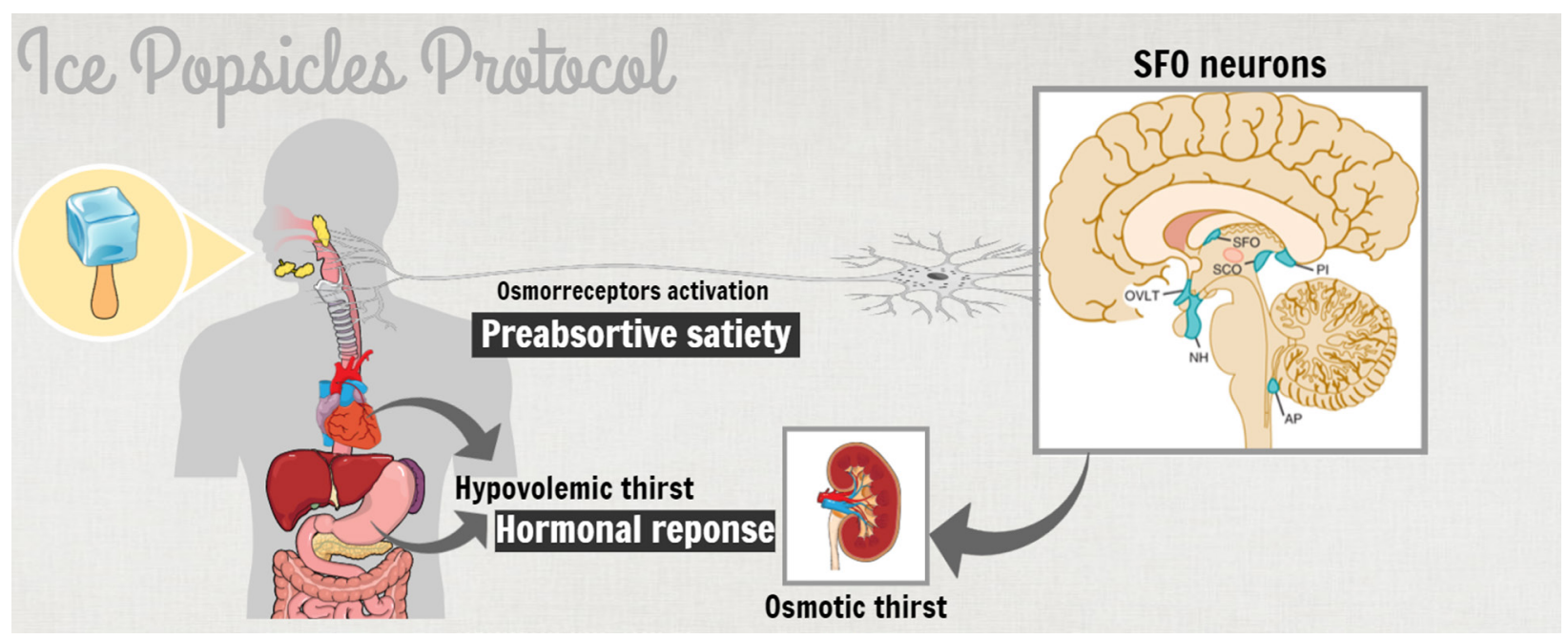

Figure 8. Schematic diagram illustrating the thirst management mechanism in postoperative patients treated with ice popsicles. The thirst relief consists of two different processes, preabsortive and hormonal response. The first is characterized by the oropharyngeal stimulation from cold and could generate thirst satiety before water consumption and changes promoted in the composition of blood. This mechanism activates the consciousness region represented by the posterior cingulate cortex, directly affected by regional cerebral blood flow, and develops an inhibition of vasopressin release. Secondly, the hormonal response is divided into two different conditions: osmotic and hypovolemic. These mechanisms occur when dehydration promotes changes in intracellular and extracellular volume. In these cases, osmotic thirst initiates with the dehydration of supraoptic neuronal cells promoted by plasma hyperosmolarity, it stimulates the secretion of the vasopressin hormone. However, hypovolemic thirst is activated with a decrease in blood volume and promotes the secretion of angiotensin II and consequently, sodium retains. All these mechanisms were activated in distinct circumstances, stimulating organs like the brain and kidneys to respond and promote the thirst relief. SFO: subfornical organ neurons. Figure created using the Mind the Graph platform.

perception. However, it may be minimized by explanation of the importance of a consistent response with the effectiveness of ice popsicles as a treatment in postoperative patients. Also, we did not consider how long the satiety and discomfort relief was maintained by the ice popsicles protocol or its preabsorptive performance, suggesting that the duration of the protocol could be maintained according to the patient's perception of discomfort. Moreover, we did not study whether the use of medications could interfere with the effect of ice popsicles. The failure to follow the previously requested recommendation of fasting time may have affected the results.

Finally, we could suggest thatice popsicles are promising to thirst management. Satisfactorily, our data showed sustained effectiveness of the ice popsicles, in concordance with the study conducted by Conchon \& Fonseca (2018). Ice popsicles has several advantages, including its low cost and easy administration, which is an essential alternative for patients with difficulty swallowing. More studies should be done to corroborate the safety and effectiveness of ice popsicles administration, considering this need and evaluating the patient's discomfort.

Considering that few protocols are currently available for immediate postoperative thirst management, the present results provided additional evidence that ice popsicles constitute a promising, innovative and simple strategy for the management of thirst, mainly after surgical procedures, adding to patient comfort and to the security of the health team that manages it (see scheme proposed in Figure 8). 


\section{Acknowledgments}

This work was supported by grants from Conselho Nacional de Desenvolvimento Científico e Tecnológico (CNPq), Coordenação de Aperfeiçoamento de Pessoal de Nivel Superior (CAPES), Fundação de Apoio à Pesquisa e Inovação do Estado de Santa Catarina (FAPESC), Programa INCT-INOVAMED (grant 465430/2014-7) and Programa de Pós-Graduação em Neurociências (PPG NEURO), all from Brazil. G.M.B. and R.S.P. are undergraduate students in Physiotherapy who received a grant from CNPq. R.C.D. is the recipient of a research productivity fellowship from the CNPq. The authors report no conflicts of interest in this work.

\section{REFERENCES}

AGRAWAL V, AGARWAL M, JOSHI SR \& GHOSH AK. 2008. Hyponatremia and hypernatremia: disorders of water balance. The J Assoc of Phys India 56: 956-964.

ALOAMAKA EO, AMABEBE E, OZOENE JO \& OBIKA LFO. 2018. Thirst perception, drinking, arginine vasopressin activity and associated neurohumoral factors. J Afr Assoc Physiol 6: 1-13.

ARAI S, STOTTS N \& PUNTILLO K. 2013. Thirst in critically ill patients: from physiology to sensation. Am Assoc Crit Care Nur 22: 328-335.

ARAI SR, BUTZLAFF A, STOTTS NA \& PUNTILLO KA. 2014. Quench the thirst: lessons from clinical thirst trials. Biol Res Nurs 16: 456-466.

ARMSTRONG LE \& JOHNSON EC. 2018. Water Intake, Water Balance, and the Elusive Daily Water Requirement. Nutrients 10: 1928-1953.

ARMSTRONG LE \& KAVOURAS SA. 2019. Thirst and Drinking Paradigms: Evolution from Single Factor Effects to Brainwide Dynamic Networks. Nutrients 11: 2864-2895.

ARONI P, FONSECA LF, CIOL MA, MARGATHO AS \& GALVAO CM. 2020. The use of mentholated popsicle to reduce thirst during preoperative fasting: A randomised controlled trial. J Clin Nurs 29: 840-851.

BOURQUE CW. 2008. Central mechanisms of osmosensation and systemic osmoregulation. Nat Rev Neurosci 9: 519-531.

BRUNSTROM JM. 2002. Effects of mouth dryness on drinking behavior and beverage acceptability. Physiol Behav 76: 423-429.

BRUNSTROM JM \& MACRAE AW. 1997. Effects of temperature and volume on measures of mouth dryness, thirst and stomach fullness in males and females. Appetite 29: 31-42.

BURY J \& ROSSENEU M. 1985. Enzyme linked immunosorbent assay for human apolipoprotein C-III. J Clin Chem Clin Biochem 23: 63-68.

CABALLERO B, ALLEN L \& PRENTICE A 2005. Encyclopedia of human nutrition. 2nd ed. / Editor-in-chief, Benjamin Caballero; editors, Lindsay Allen, Andrew Prentice. ed, Amsterdam; London: Elsevier Academic Press.

CHEUVRONT SN \& KENEFICK RW. 2014. Dehydration: physiology, assessment, and performance effects. Compr Physiol 4: 257-285.

CHO EA, KIM KH \& PARK JY. 2010. Effects of frozen gauze with normal saline and ice on thirst and oral condition of laparoscopic cholecystectomy patients: pilot study. J Korean Acad Nurs 40: 714-723.

CONCHON MF \& FONSECA LF. 2018. Efficacy of an Ice Popsicle on Thirst Management in the Immediate Postoperative Period: A Randomized Clinical Trial. Perianesth Nurs 33: 153-161.

CRITCHLEY HD \& HARRISON NA. 2013. Visceral influences on brain and behavior. Neuron 77: 624-638.

DEAUX E. 1973. Thirst satiation and the temperature of ingested water. Science 181: 1166-1167.

DENTON D, SHADE R, ZAMARIPPA F, EGAN G, BLAIR-WEST J, MCKINLEY M, LANCASTER J \& FOX P. 1999. Neuroimaging of genesis and satiation of thirst and an interoceptordriven theory of origins of primary consciousness. PNAS 96: 5304-5309.

DESSOTTE CA, RODRIGUES HF, FURUYA RK, ROSSI LA \& DANTAS RA. 2016. Stressors perceived by patients in the immediate postoperative of cardiac surgery. Rev Bras Enferm 69: 741-750.

DHAKA A, VISWANATH V \& PATAPOUTIAN A. 2006. Trp ion channels and temperature sensation. Annu Rev Neurosci 29: 135-161.

DO NASCIMENTO LA, FONSECA LF \& DOS SANTOS CB. 2018. Inter-rater Reliability Testing of the Safety Protocol for Thirst Management. J Perianesth Nurs 33: 527-536.

ECCLES R. 2000. Role of cold receptors and menthol in thirst, the drive to breathe and arousal. Appetite 34 : 29-35.

ECCLES R, DU-PLESSIS L, DOMMELS Y \& WILKINSON JE. 2013. Cold pleasure. Why we like ice drinks, ice-lollies and ice cream. Appetite 71: 357-360.

FITZSIMONS JT. 1998. Angiotensin, thirst, and sodium appetite. Physiol Rev 78: 583-686. 
GARCIA AKA, FURUYA RK, CONCHON MF, ROSSETTO EG, DANTAS RAS \& FONSECA LF. 2019. Menthol chewing gum on preoperative thirst management: randomized clinical trial. Rev Latino-Am Enferm 27: e3180.

GIZOWSKI C \& BOURQUE CW. 2017. Neurons that drive and quench thirst. Science 357: 1092-1093.

GOGOLLA N. 2017. The insular cortex. Current Biol: CB 27: R580-R586.

LABBE D, ALMIRON-ROIG E, HUDRY J, LEATHWOOD P, SCHIFFERSTEIN HN \& MARTIN N. 2009. Sensory basis of refreshing perception: role of psychophysiological factors and food experience. Physiol Behav 98: 1-9.

LATORRE R, BRAUCHI S, MADRID R \& ORIO P. 2011. A cool channel in cold transduction. Physiology 26: 273-285.

MCKINLEY MJ \& JOHNSON AK. 2004. The physiological regulation of thirst and fluid intake. News Physiol Sci 19: 1-6.

MOTTA NH, DO NASCIMENTO LA, PIEROTTII, CONCHON MF \& FONSECA LF. 2020. Evaluation of a Safety Protocol for the Management of Thirst in the Postoperative Period. J Perianesth Nurs 35: 193-197.

NASCIMENTO LA, FONSECA LF, ROSSETO EG \& SANTOS CB. 2014. Development of a safety protocol for management thirst in the immediate postoperative period. Rev Esc Enferm USP 48: 834-843.

NASCIMENTO LA, NAKAYA TG, CONCHON MF, GARCIA AKA, PIEROTTI I, SERATO VM \& FONSECA LF. 2019. Prevalence, intensity and discomfort of thirst in surgical patients in the immediate post-operative period. Revista SOBECC 24: 85-90.

NASCIMENTO LAD, GARCIA AKA, CONCHON MF, ARONI P, PIEROTTI I, MARTINS PR, NAKAYA TG \& FONSECA LF. 2020. Advances in the Management of Perioperative Patients' Thirst. AORN J 111: 165-179.

PEIER AM ET AL. 2002. A TRP channel that senses cold stimuli and menthol. Cell 108: 705-715.

PERLAS A, DAVIS L, KHAN M, MITSAKAKIS N \& CHAN VW. 2011. Gastric sonography in the fasted surgical patient: a prospective descriptive study. Anesth Analg 113: 93-97.

PEYROT DES GACHONS C, AVRILLIER J, GLEASON M, ALGARRA L, ZHANG S, MURA E, NAGAI H \& BRESLIN PA. 2016. Oral Cooling and Carbonation Increase the Perception of Drinking and Thirst Quenching in Thirsty Adults. PLoS ONE 11: e0162261.

PUNTILLO K, ARAI SR, COOPER BA, STOTTS NA \& NELSON JE. 2014. $A$ randomized clinical trial of an intervention to relieve thirst and dry mouth in intensive care unit patients. Intensive Care Med 40: 1295-1302.
PUNTILLO KA, ARAI S, COHEN NH, GROPPER MA, NEUHAUS J, PAUL SM \& MIASKOWSKI C. 2010. Symptoms experienced by intensive care unit patients at high risk of dying. Crit Care Med 38: 2155-2160.

RAMSAY DJ \& BOOTH DA 1991. Thirst: physiological and psychological aspects. London: Springer.

SAKER P, FARRELL MJ, ADIB FR, EGAN GF, MCKINLEY MJ \& DENTON DA. 2014. Regional brain responses associated with drinking water during thirst and after its satiation. PNAS 111: 5379-5384.

SALATA RA, VERBALIS JG \& ROBINSON AG. 1987. Cold water stimulation of oropharyngeal receptors in man inhibits release of vasopressin. J Clin Endocrinol metab 65: 561-567.

SALTMARSH M. 2001. Thirst: or, why do people drink? Nutr Bull 26: 53-58.

SCHAFER K, BRAUN HA \& ISENBERG C. 1986. Effect of menthol on cold receptor activity. Analysis of receptor processes. J Gen Physiol 88: 757-776.

THOMPSON CJ, EDWARDS CR \& BAYLIS PH. 1991. Osmotic and non-osmotic regulation of thirst and vasopressin secretion in patients with compulsive water drinking. Clin Endocrinol 35: 221-228.

TOSUN B, YAVA A \& ACIKEL C. 2015. Evaluating the effects of preoperative fasting and fluid limitation. Int J Nurs Pract 21: 156-165.

WANG Z, DEURENBERG P, WANG W, PIETROBELLI A, BAUMGARTNER RN \& HEYMSFIELD SB. 1999. Hydration of fat-free body mass: review and critique of a classic body-composition constant. Am J Clin Nutr 69: 833-841.

ZIMMERMAN CA, LEIB DE \& KNIGHT ZA. 2017. Neural circuits underlying thirst and fluid homeostasis. Nat Rev Neurosci 18: 459-469.

ZIMMERMAN CA, LIN YC, LEIB DE, GUO L, HUEY EL, DALY GE, CHEN Y \& KNIGHT ZA. 2016. Thirst neurons anticipate the homeostatic consequences of eating and drinking. Nature 537: 680-684.

\section{How to cite}

TEREZA DM, BALDASSO GM, PAES RS, PACHECO MEJ, ROSA PPS, VENDRAMINE B, HULSE SC, CAPASSO R \& DUTRA RC. 2021. Evaluation of the protocol for thirst management using ice popsicles in the immediate postoperative period: A pilot study in southern Brazilian hospital. An Acad Bras Cienc 93: e20201260. DOI 10.1590/0001-3765202120201260.

Manuscript received on August 7, 2020; accepted for publication on October 30, 2020 
DENISE M. TEREZA ${ }^{1,2}$

https://orcid.org/0000-0003-0531-8360

\section{GABRIELA M. BALDASSO ${ }^{1}$}

https://orcid.org/0000-0002-6254-2812

\section{RODRIGO S. PAES ${ }^{1}$}

https://orcid.org/0000-0002-2330-4862

\section{MARIA E.J. PACHECO ${ }^{1}$}

https://orcid.org/0000-0002-8834-6916

\section{PATRÍCIA P.S. ROSA ${ }^{3}$}

https://orcid.org/0000-0002-7696-8734

\section{BEATRIZ VENDRAMINE ${ }^{4}$}

https://orcid.org/0000-0001-9590-1538

\section{SERGIO C. HULSE ${ }^{4}$}

https://orcid.org/0000-0002-7738-0158

\section{RAFFAELE CAPASSO}

https://orcid.org/0000-0002-3335-1822

\section{RAFAEL C. DUTRA ${ }^{1,2}$}

https://orcid.org/0000-0002-6938-2161

${ }^{1}$ Universidade Federal de Santa Catarina, Departamento de Ciências da Saúde, Laboratório de Autoimunidade e Imunofarmacologia, Campus Araranguá, Rodovia Governador Jorge Lacerda, 3201, 88906-072 Araranguá, SC, Brazil

${ }^{2}$ Programa de Pós-Graduação em Neurociências, Centro de Ciências Biológicas, Universidade Federal de Santa Catarina, Rua Engenheiro Agronômico Andrei Cristian Ferreira, s/n, 88040-900 Florianópolis, SC, Brazil
${ }^{3}$ Programa de Pós-Graduação em Ciências da Reabilitação, Campus Araranguá, Universidade Federal de Santa Catarina, Rua Pedro João Pereira, 150, 88906-072 Araranguá, SC, Brazil

${ }^{4}$ Hospital Unimed, Avenida Estevão Emílio de Souza, 101, 88815-180 Criciúma, SC, Brazil

${ }^{5}$ Departamento de Ciências Agrárias, Universidade de Nápoles Federico II, Rua Corso Umberto I, 40, 80055 Portici, Itália

Correspondence to: Rafael Cypriano Dutra

E-mail: rafaelcdutra@gmail.com; rafael.dutra@ufsc.br

\section{Author contributions}

Conceptualization, D.M.T., G.M.B. and R.C.D.; methodology, D.M.T., G.M.B., R.S.P., M.E.J.P., P.P.S.R., B.V., S.C.H. and R.C.D.; formal analysis and funding acquisition, D.M.T. and R.C.D., analyzed the data, D.M.T., G.M.B., R.S.P., M.E.J.P., P.P.S.R., B.V., S.C.H. and R.C.D.; writing-original draft preparation, D.M.T., G.M.B., R.S.P., R.C. and R.C.D.; writing-review and editing, G.M.B., R.C. and R.C.D.; supervision, R.C.D. All authors have read and agreed to the published version of the manuscript.

\section{(cc) BY}

